•综述・

\title{
植物群落稀有种维持机制与土壤反馈的研究进展
}

\author{
戴冬@1,2，邢华@1,2，杨佳线 ${ }^{1,2}$, 刘雅静 1,2 , 蔡焕满 ${ }^{3}$, 刘宇@1,2,4*
}

1. 华东师范大学-阿尔伯塔大学生物多样性联合实验室, 华东师范大学生态与环境科学学院, 上海 200241; 2. 浙江天童森林生态系统国家 野外科学观测研究站, 浙江宁波 315114３. 凤阳山-百山祖国家级自然保护区管理局百山祖管理处，浙江丽水 323808; 4. 上海污染控制与 生态安全研究院, 上海 200092

摘要: 自Janzen-Connell (J-C)假说提出后半个世纪以来, 生态学家在热带及亚热带森林对该假说开展的大量实证研究表明, 由专性天敌导致的J-C效应所引起的负密度制约是维持森林多样性和决定群落组成的重要驱动力, 该假说成功地解释了热带 及亚热带森林的丰富多样性。土壤病原真菌所引起的植物-土壤负反馈是J-C效应最主要的表现形式。然而，对于植物-土壤 负反馈是否能够维持森林群落中的大量稀有种仍然存在许多争议。基于当代物种共存理论的“稀有种优势”假说认为, 只有在 满足 “可入侵准则” (即物种在稀有时具有种群增加的趋势)的前提下, 稀有种才能在群落中与其他物种长期共存。然而, 当前 基于土壤反馈的实验结果与该理论预测相悖，因此在稀有种的维持机制方面仍存在较大的分歧。本文通过介绍植物-土壤反 馈理论, 整合了可能对稀有种维持有较大影响的因素, 包括共生菌根真菌、土壤养分以及植物细根性状等在影响土壤负反馈 方面的相关研究, 并对这些因素如何影响群落中物种多度和稀有种在群落中的维持进行了探讨。最后, 我们也从其他角度探 讨了一些对稀有种维持的研究。我们认为在未来对稀有种的研究中，探讨使其长期存续的“优势”和制约其种群扩大的“限制” 同等重要, 将当代物种共存理论与新技术、新方法相结合对于探究稀有种的维持机制具有重要的意义, 可为稀有种保护提供 理论依据。

关键词: 同种负密度制约; 森林群落; 入侵准则; Janzen-Connell假说; 植物-土壤反馈; 稀有种; 物种多度

戴冬, 邢华, 杨佳线, 刘雅静, 蔡焕满, 刘宇 (2021) 植物群落稀有种维持机制与土壤反馈的研究进展. 生物多样性, 29, 1687-1699. doi: 10.17520/biods.2021141.

Dai D, Xing H, Yang JR, Liu YJ, Cai HM, Liu Y (2021) Advances in mechanisms of rare species maintenance and plant-soil feedback in plant communities. Biodiversity Science, 29, 1687-1699. doi: 10.17520/biods.2021141.

\section{Advances in mechanisms of rare species maintenance and plant-soil feedback in plant communities}

Dong Dai ${ }^{(1,2}$, Hua Xing ${ }^{1,2}$, Jiarong Yang ${ }^{1,2}$, Yajing Liu ${ }^{1,2}$, Huanman $\mathrm{Cai}^{3}$, Yu Liu ${ }^{\left(1,2,4^{*}\right.}$

1 ECNU-UAlberta Joint Lab for Biodiversity Study, School of Ecology and Environmental Sciences, East China Normal University, Shanghai 200241

2 Tiantong National Station for Forest Ecosystem Research, Ningbo, Zhejiang 315114

3 Management of Baishanzu, Fengyangshan-Baishanzu National Nature Reserve, Lishui, Zhejiang 323808

4 Shanghai Institute of Pollution Control and Ecological Security, Shanghai 200092

\section{ABSTRACT}

Background \& Aim: Since the Janzen-Connell (J-C) hypothesis was proposed half a century ago, a mounting number of studies have been conducted to test the hypothesis in tropical and subtropical forests. These studies have since greatly improved our understanding of how high biodiversity is maintained. In particular, the pathogenic fungi-induced J-C effect, a type of negative plant-soil feedback (PSF), has been well-recognized as a mechanism to maintain biodiversity and structure community composition, though the overall contribution of PSF to the persistence of a large number of rare species in nature remains controversial. As predicted by the modern species coexistence theory, the "invasion criterion" should be met for rare species to co-exist with other species such that one species will increase in abundance when rare. However, previous studies show results contrary to the prediction of such theory and have thus sparked

收稿日期: 2021-04-14; 接受日期: 2021-07-20

基金项目: 国家自然科学基金(31670531; 32071645)

* 通讯作者 Author for correspondence. E-mail: yuliu@des.ecnu.edu.cn 
debates on the mechanism underlying rare species maintenance.

Progresses: In this work, we review PSF and the potential factors associated with PSF, including mycorrhizal fungi, soil nutrient content, and fine root functional traits. We discuss their contributions in maintaining rare species and determining species abundance via PSF. In addition to PSF, some other perspectives about rare species maintenance are also covered in this review.

Prospects: We propose that the advantages in maintaining the long persistence of rare species and the limitations in restricting population expansion of rare species may be of equal importance for rare species. The combination of modern species coexistence theory and new techniques and methodologies provide promising future directions to fully understand rare species and to better conserve rare species in the future.

Key words: conspecific negative density dependence; forest community; invasion criterion; Janzen-Connell hypothesis; plant-soil feedback; rare species; species abundance

热带和亚热带森林因具有较高的生物多样性 而受到生态学家的关注, 研究其多样性的维持机制 一直是生态学的核心问题之一。这些具有高多样性 的森林最典型的特征就是存在大量的稀有树种。以 2015年巴拿马Barro Colorado Island (BCI) 50 ha热 带森林动态监测样地的复查数据为例, 尽管稀有树 种的个体总数仅占样地内 229,000 株个体的 $0.76 \%$, 但样地内 300 多个树种中稀有种多达 $40 \%$ (图1)。此 处以Hubbell和Foster (1986)基于大样地的稀有种定 义为依据来计算稀有种比例, 即每公顷平均物种多 度 $\leq 1$ 株 $(\mathrm{DBH} \geq 1 \mathrm{~cm})$ 的个体。但在不同条件下，稀

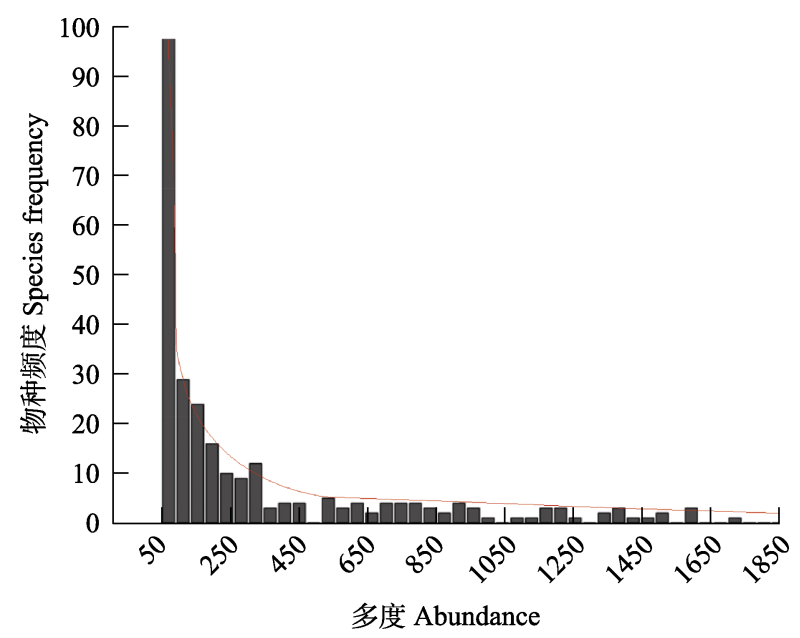

图1 Barro Colorado Island (BCI) 50 ha热带森林动态监测 样地物种频度与物种多度的关系。曲线为趋势线。每个直方 图宽度表示的多度跨度为50 (如第一个直方为多度 1-50; 由 于图幅限制, 多度显示不完全); 数据来源: BCI样地2015年 复查数据。

Fig. 1 The relationship between tree species frequency and species abundance in Barro Colorado Island (BCI) 50 ha tropical forest dynamics plot. The curve is the trend line. Width of each bar represents abundance span for 50 (e.g. the fist bar for the abundance from 1 to 50 ; the abundance is not complete due to the limitation of the picture size).
有种的定义也并不绝对, 如Rabinowitz (1981)就从 不同角度指出了植物的7种稀有形式。在全球尺度 上, Enquist等(2019)的研究甚至发现, 地球上约 435,000种已知的植物中多达 $36.5 \%$ 是极度稀有种。

早在20世纪60年代初, 生态学家已开始思考稀 有种与常见种的共存问题(Preston, 1962), 如自然界 中为何存在如此多的稀有种? 它们又是如何在自 然界中持久存在的? 尽管如此，早期对植物多样性 的研究仍更多地关注森林中的常见种, 而对稀有种 的关注较少。近年来, 随着植物谱系多样性和功能 多样性研究的发展, 稀有种在自然群落中的作用越 来越受到关注(Mi et al, 2012, 2021; Jain et al, 2014; Leitão et al, 2016)。

引起物种稀有的原因多种多样, 既可能是由植 物自身的因素引起, 如自身适合度较低(Leimu et al, 2006), 也可能是来自外部的因素所导致, 如生境破 坏、气候变化等(He, 2009)。整体而言稀有种都具有 种群小和分布区狭窄等特征(Chapman et al, 2018), 因此它们在面临环境变化时(如人类活动引起的生 境破碎化、气候变暖等)有更高的灭绝风险(Pimm et al, 1988, 1995; Smith \& Knapp, 2003; Jain et al, 2014), 更易走向灭绝漩浴 (extinction vortex; Caughley, 1994)。群落中大量稀有种的存在显著提高了群落的 $\alpha$ 多样性, 同时对生态系统功能也有非常重要的作 用(Le Bagousse-Pinguet et al, 2021)。因比，保护稀有 种是生物多样性保护的重要内容, 探讨稀有种的维 持机制对于生物多样性保护和解释物种共存具有 重要的意义。

近年来快速发展的植物-土壤反馈 (plant-soil feedback, PSF)理论为解释稀有物种在群落中的共 存与森林树种多度差异提供了一个突破点。Janzen 
和Connell于 20世纪70年代初期各自独立提出了天 敌引起的宿主密度/距离制约效应, 即 Janzen-Connell (J-C)假说, 以解释热带森林的高度 多样性(Janzen, 1970; Connell, 1971)。在过去的半个 世纪J-C假说得到了极大的发展: 不仅在热带森林 中得到广泛验证(Augspurger \& Kelly, 1984; Bell et al, 2006; Li et al, 2009; Mangan et al, 2010b; Bagchi et al, 2014), 还在亚热带森林(Liu et al, 2012; Liang et al, 2015)、温带森林(Packer \& Clay, 2000; Jia et al, 2020)以及草地生态系统(Petermann et al, 2008)得到 了实证支持。随着对 J-C假说研究的不断深入, 植食 性昆虫和动物以及植物病原菌被发现是这一效应 的主要驱动力。其中, 土壤病原真菌因具有较高的 宿主专性、快速增殖的能力及对植物的种子和幼苗 阶段较大的杀伤力而受到广泛关注, 并被大量研究 证实是导致J-C效应的主要驱动因素(Packer \& Clay, 2000; Bell et al, 2006; Liu et al, 2012; Bagchi et al, 2014; Jia et al, 2020)。因此, 研究土壤病原真菌所引 起的土壤负反馈强度的差异有助于理解自然群落 中植物多度差异的形成机制 (Klironomos，2002; Mangan et al, 2010b; Liu et al, 2015; Liang et al, 2016)。

本文围绕当前稀有种维持机制方面的各种观 点, 结合当代物种共存理论来解释稀有种优势假说, 并介绍了当前植物一土壤反馈理论及其在稀有种维 持方面的研究进展和存在的争议, 以期为更深入地 探讨稀有种维持机制提供新的视角。

\section{稀有种维持与稀有种优势假说}

\section{1 经典物种共存理论与稀有种维持}

森林木本植物群落可以看成是常见种、中间种 (多度介于常见种和稀有种之间的物种, intermediate species)和稀有种共存的群落。经典的物种共存理论 强调生态位的分化，例如不同物种在资源、时间以 及空间等方面的分化(Grinnell, 1917)。长期以来，生 态位分化这一经典理论在研究群落生物多样性维 持方面一直占据主导地位, 学界也普遍认可生态位 分化对物种共存的重要性。根据传统的生态位理论, 有学者认为稀有种的稀有性是由于与其他竞争者 的生态位有重叠, 而稀有种竞争能力较弱所导致 (Dawson et al, 2012)。但如果稀有种竞争能力较弱, 并且处于多度的弱势地位, 那么应该更容易被竞争
排除，然而事实上稀有种仍然大量存在，而且在一 些研究中发现稀有种的竞争能力并不弱于常见种 (Lloyd et al, 2002; Zhang \& van Kleunen, 2019)。此 外, 也有一些学者认为稀有种之所以稀有, 可能是 因为生态位分化使其占据着特殊生态位(Brown, 1984; Gaston et al, 1997; Yenni et al, 2017)。这一观点 虽然被一些研究所验证(Ai et al, 2013; Mi et al, 2021), 但由于量化群落中大量个体的生态位宽度 和性状变异的工作量巨大，仍缺乏更为直接与广泛 的证据。因此, 传统生态位理论在解释稀有种的稀 有机制方面存在一定的局限性，而当代物种共存理 论的提出则为解释这一机制提供了新的思路。

\section{2 当代物种共存理论与稀有种优势假说}

当代物种共存理论由Chesson于 2000年提出, 该理论将物种间的差异划分为两种类型，即生态位 差异 (niche difference) 和平均适合度差异 (fitness difference), 储诚进等(2017)对这一理论进行了详细 的介绍。简而言之, 生态位差异与传统的物种共存 理论相近，强调时间、空间和资源等方面的分化, 而平均适合度差异则强调物种在资源利用、对抗天 敌和生长繁殖等方面的差异, 也可以理解为竞争能 力的差异。物种间的平均适合度差异越大越难以共 存，因为适合度高的物种总是倾向于将适合度低的 物种竞争排除出群落。因此, 群落中的物种一方面 可通过提高生态位差异来避免生态位重叠, 即稳定 化机制(stabilizing mechanism)来实现共存, 另一方 面也可以通过降低平均适合度差异，减少将对方排 除, 即均等化机制(equalizing mechanism)来实现共 存(储诚进等, 2017)。当代物种共存理论为物种共存 研究提供了一个具有综合性和普适性的理论框架。

根据当代物种共存理论，当一个物种入侵一个 由竞争者建立的稳定群落时, 要经历正的种群增长, 由此导致物种在稀有时的优势(MacArthur \& Levins, 1967; Chesson, 2000), 即 “稀有种优势” 假说 (Siepielski \& McPeek, 2010)。该假说是稀有种维持 机制的一个重要依据。早在1984年, Connell等在研 究热带森林中的树种共存时就认为稀有种只有具 有某种优势时才能防止局部灭绝并维持整个群落 的高度多样性，他们将该优势称作“群落补偿趋势” (community compensatory trend, Connell et al, 1984)。当一个物种稀有时, 其种群的平均适合度增 加, 使得种群多度增长, 种群得以恢复; 当一个物 
种常见时, 其平均适合度降低, 从而阻止种群规模 的进一步增加, 使得稀有种获益, 这一过程也是稳 定化机制的体现(Chesson, 2000; 储诚进等, 2017)。

这种物种在稀有时种群多度增加的趋势也被生态 学家称作“可入侵准则(invasion criterion)”, 该准则 同时也是衡量几个物种是否处于共存状态的重要 标准: 如果群落中的所有种都满足当其数量稀有时 种群可增长的条件, 才能说它们是处于共存的状态 (MacArthur, 1972; Chesson, 2000; Adler et al, 2007)。Grainger等(2019)甚至认为这一准则是生态 学研究中的“通用货币”。尽管得到广泛的理论支持, 但“稀有种优势”假说在自然环境下难以验证, 因 此存在一定的争议。

\section{2 土壤反馈与稀有种维持}

\section{1 从 J-C假说到土壤反馈}

在 Janzen (1970)和Connell (1971)提出 JanzenConnell假说来解释热带森林中的同种负密度制约 (conspecific negative density dependence, CNDD)现 象之后, 大量的研究从各个角度对这一假说进行了 论证。J-C假说认为, 母树的种子以母树为中心扩散, 离母树越远种子数量越少, 离母树越近幼苗遇到专 性天敌的可能性越高, 幼苗的死亡率也越高, 这一 机制维持了热带及亚热带森林中物种的多样性。通 过实验发现, 许多生物都能够通过对植物的专性取 食或寄生引起J-C效应, 如植食性昆虫(Forrister et al, 2019)、叶片病原菌(Bayandala et al, 2017)和土壤病 原菌(Augspurger, 1984; Packer \& Clay, 2000; Bell et al, 2006; Petermann et al, 2008)等。祝燕等(2009)在 对负密度制约的综述中有过详细介绍。应当指出的 是, 专性天敌所引起的J-C效应只是引起CNDD的 一部分因素。除J-C效应之外, 相似资源的竞争以及 化感作用等也可以引起CNDD。虽然目前普遍认为 专性天敌是CNDD的最主要来源, 但二者并不能完 全等同。近年来的大量研究发现, 土壤病原菌产生 的土壤负反馈引起的J-C效应是维持森林树种多样 性的最主要驱动因素(Packer \& Clay, 2000; Mangan et al, 2010b; Liu et al, 2012; Jia et al, 2020)。具有较 高宿主专性的土壤病原菌因对植物的种子萌发、幼 苗定植和生长这些对种群结构非常重要但相对较 为脆弱的阶段有较强的负面影响, 从而对同种植物 产生较强的负反馈作用。
然而, 虽然许多实验验证了 J-C假说, 并通过换 土、施用杀菌剂等实验证明了土壤病原菌在其中扮 演的重要角色, 但是仍然有许多实验并没有检测出 土壤负反馈。Hyatt等(2003)通过一项综合了许多控 制性实验的荟萃分析发现, 距离制约引起的J-C效 应在自然界并不是一个普遍的现象。与此同时, 越 来越多的研究发现除了土壤病原菌之外, 以丛枝菌 根 (arbuscular mycorrhizal, AM) 真菌、外生菌根 (ectomycorrhizal, EM) 真菌为主的植物共生真菌由 于可以通过增强植物的养分吸收、产生物理或化学 防护等方式产生土壤正反馈, 从而削弱了土壤负反 馈, 这很可能是导致在之前的许多实验中无法检测 到负反馈或负反馈在不同研究中差异较大的原因。 外生菌根真菌可以通过形成菌根套来阻碍病原菌 侵染植物细根, 从而显著降低外生菌根植物受到的 土壤负反馈作用(Marx, 1972); 而从枝菌根真菌则 可以通过诱导寄主产生一系列的免疫反应来提升 宿主对病原菌的抗性(Jung et al, 2012; Cameron et al, 2013)。可见, 无论是外生菌根真菌还是丛枝菌根真 菌, 在与细根形成共生后都能在不同程度上降低土 壤病原真菌对植物细根的侵害。通过湿篮法分离从 枝菌根真菌孢子和病原真菌后进行的接种实验进 一步发现, 从枝菌根真菌和病原菌在母树下都存在 距离依赖, 并且丛枝菌根真菌可以抵消土壤病原菌 引起的J-C效应(Liang et al, 2015); 而对长期幼苗数 据及土壤真菌群落进行测序分析发现, 不同物种的 负反馈强度差异主要受菌根类型以及各类根际土 壤真菌的积累速率的调控(Chen et al, 2019)。这些 证 据都表明菌根真菌影响了土壤病原菌引起的负反 馈, 并可以在某种程度上影响群落结构。由于绝大 部分植物都会与从枝菌根或外生菌根等真菌形成 共生(Smith \& Read, 1997; Brundrett, 2009), 因此在 考虑由土壤病原菌引起的负反馈时不能忽视菌根 真菌产生的正反馈作用。土壤病原菌引起的负反馈 与菌根真菌引起的正反馈之和被称作植物-土壤净 反馈能力。植物一土壤净反馈通常作为直观反映土 壤反馈强度的综合体现(Liu \& He, 2019)。

\section{2 土壤反馈与物种多度}

土壤反馈理论对于解释热带森林多样性具有 重要的作用, 因此可以结合稀有种由于其密度低可 逃避专化性天敌的侵害(Vermeij \& Grosberg, 2018), 同时依据“可入侵准则” (Siepielski \& McPeek, 2010; 
Grainger et al, 2019)和天敌逃逸假说 (Keane \& Crawley, 2002), 并基于当代物种共存理论的入侵生 态学框架(于文波和黎绍鹏, 2020), 尝试从土壤反馈 理论促进物种共存的角度入手, 解析稀有种的存在 及维持机制。

根据“稀有种优势”假说, 在群落中稳定存在的 稀有种种群应具有较强的增长趋势, 而常见种的种 群增长则受到更强的阻力。如果稀有种优势来自植 物-土壤反馈, 那么稀有种理论上应该受到较弱的 土壤净负反馈才有利于种群增长, 而常见种应当受 到较强的土壤净负反馈, 其种群的进一步增长受到 抑制。简而言之, 理论上土壤净负反馈强度应与物 种的多度呈正相关。例如, 在一些草地生态系统和 弃耕农田中对草本植物的研究发现了稀有种或低 密度种受负反馈更低的现象 (van de Voorde et al, 2012; Kos et al, 2013; Maron et al, 2016)。在森林生 态系统中, Liu等(2015)在亚热带森林中通过幼苗实 验对光叶红豆(Ormosia glaberrima)的高密度种群和 低密度种群所受到的土壤负反馈进行对比, 发现光 叶红豆幼苗在高密度种群的土壤中受到的土壤负 反馈更强, 而在低密度种群的土壤中受到的土壤负 反馈较弱。Schroeder等(2018)在墨西哥的热带森林 中通过对根际土壤真菌进行高通量测序的方法对 常见种和稀有种根际真菌群落进行了分析, 发现树 种多度与根际共生真菌和病原菌的多样性均呈负 相关, 但与共生真菌的负相关更强, 这一结果暗示 稀有种在与土壤真菌群落的相互作用方面可能存 在优势。

以上研究均发现低密度种群在植物-土壤反馈 方面存在优势, 其幼苗受到更低的土壤负反馈, 从 而相对于高密度种群有更强的种群增长潜力。总体 而言, 由于木本植物的研究难度高于草本植物, 因 此在森林生态系统中这一结论还有待进一步验证。

然而, 也有研究得出了与“稀有种优势假说” 相 反的结论。Klironomos (2002)对比本地稀有种和入 侵种所受的负反馈差异后发现, 稀有种在同种土壤 中的生物量因受到来自土壤病原菌的胁迫而下降, 而入侵种则在同种土壤中通过菌根真菌而受益; 植 物积累病原菌的速度可以决定该物种在多大密度 时才受到负反馈, 进而影响物种在群落中的多度。 这一结果首先明确否定了 “可入侵准则”, 即稀有种 虽然与入侵种刚入侵时类似, 都具有较低的种群密
度, 但是它们的植物一土壤反馈模式却截然不同: 稀有种并不具有类似入侵种的优势。此外, Klironomos (2002)认为稀有种之所以稀有是因为其 更容易积累专性病原菌, 因而在较低密度时即可表 现出土壤负反馈, 而常见种可以达到更大密度是因 为其专性病原菌积累缓慢, 只有达到较高密度时才 会表现出负反馈。

此后, Mangan等(2010b)在BCI和Gigante的热带 森林中通过控制性实验发现, 母树的相对多度与土 壤负反馈呈负相关, 即相对多度越高的树种其幼苗 所受的土壤负反馈越小, 而这种负反馈差异是导致 常见种之所以常见, 稀有种之所以稀有的主要原 因。Klironomos (2002)和Mangan等(2010b)的观点一 致, 都认为多度与负反馈强度呈负相关, 稀有种受 到更强的负反馈。另外, Comita等(2010)和Johnson 等(2012)也通过 “从格局推断过程”的方式得出了稀 有种受更强的同种负密度制约效应的结论。其中, Comita等(2010)使用层级贝叶斯法对BCI样地中的 幼苗数据进行分析, 通过测试物种对邻近个体的敏 感性与其在群落中的相对多度的关系, 发现稀有种 比常见种受到更强的同种负密度制约; Johnson等 (2012)则使用美国森林清查数据验证了物种局域多 度与负密度制约的关系, 同样得出了稀有种受更强 负反馈的结论。然而, 这种“从格局推断过程”的思 路并不是为了探讨稀有种持续存在的原因, 而是用 这一现象来解释分析结果, 因此其结论是否可靠值 得商榷。

稀有种由于种群小, 已经在群落尺度上处于濒 临灭绝的边缘, 如果受到更强的土壤负反馈则显然 更难满足“物种在稀有时具有种群恢复的趋势”这一 物种共存的基本条件(Chesson, 2000, 2008; Adler et al, 2007)。一些研究者也在相关研究中得出了稀有 种受更强负反馈的类似结论(MacDougall et al, 2011; Yenni et al, 2012, 2017; Kempel et al, 2018)。如Yenni 等(2012, 2017)通过理论模型研究发现, 稀有种受 到的强自我限制能力促进了稀有种的长期存续, 强自我限制是稀有种存续的必要条件。

此外, 还有一些研究人员尝试从其他角度去寻 找稀有种优势, 或者试图解释稀有种受更强负反馈 的实验结果与稀有种在生态系统中有大量存在的 矛盾。Bachelot和Kobe (2013)对热带森林中植物受 到的天敌伤害类型进行研究发现, 天敌造成伤害类 
型的多样性随物种多度的增加而增加, 而天敌造成 伤害类型的多样性越高, 植物的死亡风险越高, 这 表明常见种会面临更多的天敌, 从而解释了稀有种 在躲避天敌方面具有优势。Parker等(2015)从谱系的 角度出发, 认为大多数病原体可以感染一种以上的 物种, 导致病原体溢出到密切相关的其他物种上, 这可能导致土壤负反馈强度与宿主的多度不相关。 结合系统发育模型, 他们认为稀有种在系统发育方 面拥有优势。Schroeder等(2020)认为, 常见种可能 受到较小的负反馈并不是因为其受到较低的同种 土壤负反馈, 而是受到更高的异种土壤负反馈, 这 从研究方法的角度对稀有种受到的负反馈强度与 理论存在差异进行了解释。

尽管存在许多争议, 一些坚持稀有种劣势观点 的学者仍在通过各种方式试图从其他研究角度来 证实或解释这一观点。一些学者从基因的角度研究 了稀有种稀有的原因(Marden et al, 2017; Stump et al, 2020)。Marden等(2017)通过检测热带地区6个物 种的幼苗根部发现, 较小的种群总体上具有较低的 $\mathrm{R}$ 基因(resistance gene, 病原抗性基因)氨基酸多样 性。他们认为稀有种较小的种群降低了 $\mathrm{R}$ 基因多样 性和基于病原菌识别的免疫反应, 从而导致稀有种 的种群有更强的病害传播能力和更强的易感性; 如 果局域尺度的稀有种种群无法从更大的外部种群 获得新的 $\mathrm{R}$ 基因等位基因来增强防御, 则本地稀有 种将无法维持其多度并将走向灭绝。文章的结论否 定了稀有种优势。这一结论也同时否定了稀有种与 常见种在生态系统中处于长期共存状态这一基本 假设。

综上, 大量相关研究已经证明土壤负反馈是产 生负密度制约从而维持森林多样性的重要驱动因 素, 但在物种多度与负反馈强度的关系上还存在相 当大的争议。尽管如此, 土壤微生物与植物的相互 作用仍被认为是解释森林生态系统的多样性机制 以及稀有种在群落中存续的关键因素。

\section{3 调控土壤负反馈的其他因素}

\section{1 植物与菌根真菌的共生关系}

研究人员很早就意识到与植物共生的菌根真 菌可以通过产生正反馈来抵消部分病原菌引起的 负反馈(Marx, 1972; Newsham et al, 1995; Wehner et al, 2010), 但因为菌根真菌在土壤中广泛存在, 且
绝大部分植物均会与菌根真菌形成共生, 但并不像 植物一病原菌那样存在较强的种水平上的专性共生, 所以认为菌根真菌只是广泛地对整个植物群落施 加正反馈, 而对菌根真菌是否可以影响群落结构并 不了解。随着研究的深入, 不同从枝菌根真菌物种 或群落对宿主植物具有不同作用的现象被逐渐发 现, 因此其对群落水平物种多样性的维持能力的影 响也逐渐为生态学家所关注(van der Heijden et al, 1998; Bever, 1999)。研究人员发现不同植物物种对 丛枝菌根真菌的依赖程度不同, 对接种不同丛枝菌 根真菌的响应也不同(van der Heijden et al, 1998)。 通过改变从枝菌根真菌的群落组成, 可对植物生长 产生由正到负的影响 (Bever, 2002; Klironomos, 2003)。此外, 有的植物会改变自身土壤中的丛枝菌 根真菌群落, 使之对其后代更有益, 而同时对异种 植物不利(Bever, 2002; Mangan et al, 2010a)。而在稀 有种的维持方面, Bachelot等(2017)在群落尺度上发 现, 丛枝菌根真菌多样性会使稀有种受益, Schroeder等(2020)通过计算机模拟的研究也支持菌 根真菌的宿主亲和力会影响群落结构。这些证据表 明, 从枝菌根真菌并不是简单地对所有植物施加相 同的正反馈, 而是随着植物种类、多样性的差异而 存在巨大差异。相同从枝菌根真菌对不同宿主植物 也会存在亲和力的差异。从而导致其具有影响群落 结构的能力, 并可能对稀有种的维持产生潜在的影 响。但受限于土壤从枝菌根真菌的鉴定、分离和培 养手段, 宿主植物与从枝菌根真菌的亲和力差异如 何影响群落的物种多度差异还缺乏更具体的证据。

外生菌根真菌的宿主种类远少于从枝菌根真 菌。据统计, 外生菌根真菌仅可以和部分裸子植物, 以及壳斗科、桃金娘科、龙脑香科和木麻黄科等一 些植物形成互利共生的关系。相比于从枝菌根真菌, 外生菌根真菌不仅可以增强植物的养分吸收能力 (Rousseau et al, 1994; Plassard \& Dell, 2010), 还可 以通过形成菌根鞘提供物理防护、释放抗菌化合物 等方式帮助植物抵御根部病原微生物的侵害, 来保 护植物细根(Marx, 1972), 从而显著地降低土壤病 原菌引起的负反馈(Chen et al, 2019)。因为外生菌根 真菌对宿主植物根系有较强的保护作用, 所以被 认为可以促进单科或单种优势森林植被的形成, 对多样性的维持不利(Hart et al, 1989; Corrales et al, 2016)。近期, 邢华等(未发表数据)通过对浙江省庆 
元县百山祖国家公园中的极度濒危树种百山祖冷 杉(Abies beshanzuensis)的根际微生物进行研究发现, 百山祖冷杉的细根与外生菌根真菌高度共生(外生 菌根真菌相对丰度显著高于其他两种冷杉), 且其 外生菌根真菌的物种组成不同于其他外生菌根真 菌植物及其他两种非稀有冷杉。因此这种对专性菌 根真菌的高度依赖可能反而限制了百山祖冷杉种 群在母树以外的其他地方的增长。这一发现表明, 一些稀有种确实从土壤微生物中获得了优势, 但宿 主与外生菌根真菌之间的专性共生可能又会限制 种群增长, 即百山祖冷杉的外生菌根真菌在保证宿 主稀有种优势的同时又限制了宿主的种群规模。以 往对外生菌根真菌与植物群落多样性方面的认识 多在其如何帮助植物形成单种优势并降低多样性 方面(McGuire, 2007; Corrales et al, 2016), 但在群落 水平上外生菌根真菌的扩散限制是否会影响外生 菌根树种的分布, 并进而影响群落的多样性还有待 研究。未来可以尝试通过分析其他外生菌根树种的 菌根真菌与宿主的依赖性来探索外生菌根真菌专 性对宿主种群分布的影响。

\section{2 细根性状、养分与土壤反馈}

植物细根是植物对养分、水分吸收的最末端结 构(Pregitzer, 2002), 同时也与土壤中的微生物直接 进行相互作用。许多研究发现植物细根性状的变异 从资源进取型至资源保守型变化 (Reich, 2014; Kramer-Walter et al, 2016), 这也被生态学家称作根 经济谱(root economics spectrum)。植物的细根性状 会随着其所在土壤养分含量的变化而快速周转 (turnover)和变化(Ho et al, 2005)。例如, 植物在土壤 养分低时可能会通过产生直径更小、比根长 (specific root length, SRL)和比根面积(specific root area, SRA)更大的细根之类的方式来提高单位碳投 入所能探索的土壤体积范围(Lynch \& Ho, 2005), 而 更大的比根长和比根面积也有利于养分吸收和菌 根真菌的侵染(Guo et al, 2008; McCormack et al, 2015; Cortois et al, 2016)。因此, 由于土壤养分变化 引起的细根性状响应变化可能也会影响细根对病 原微生物或恶劣环境的抗性 (Eissenstat, 1992; Newsham et al, 1995)。在西澳大利亚的研究发现, 极度缺乏磷元素的土地上往往具有极高的物种多 样性, 如澳大利亚西部Kwongan的 0.01 ha内有 40 个 灌木树种, 并且各个种均存在成年个体(Laliberté et al, 2015)。这可能是因为土壤极端缺乏磷元素导致 植物的细根更加倾向于在较低投入情况下探索更 多的土壤, 从而导致细根更易感病, 植物更容易受 到负密度制约(Laliberté et al, 2012, 2015)。在对澳大 利亚西部的Jurien Bay自然形成的连续时间序列土 壤进行研究后发现, 随着生态系统的发展, 土壤中 的无机磷含量逐渐减少, 植物的养分吸收策略也逐 渐多样化, 植物的多样性随着土壤有效磷的降低而 增加(Zemunik et al, 2015), 这也进一步说明了土壤 磷含量可以通过塑造细根养分获取策略进而影响 植物群落多样性。

相对于在贫㾑土壤上较为一致的实验结论, 一 些研究也对养分添加如何影响植物土壤反馈进行 了探讨, 但是结论并不相同。一些研究发现, 养分 添加会降低土壤负反馈(Gustafson \& Casper, 2004; in’t Zandt et al, 2019), 主要原因是养分添加缓解了 植物受到的养分胁迫, 从而可以对病原菌产生更强 的耐受力; 而另一些研究则发现在养分充足的环境 下, 植物会降低对菌根真菌的依赖(Balzergue et al, 2011; Kobae et al, 2016; Regus et al, 2017), 这是因 为与菌根真菌的共生需要消耗植物合成的碳水化 合物, 反而会成为植物的负担, 这会使其与真菌的 共生关系逐渐丧失, 最后导致土壤病原菌对植物造 成更大的威胁(Porter \& Sachs, 2020)。基于分子水平 的研究发现, 磷酸盐也是调节从枝菌根真菌定植的 信号分子。当根系磷酸盐浓度高时, 可以抑制植物 的磷酸盐饥饿信号对丛枝菌根真菌定植的诱导 (Branscheid et al, 2010)。可见, 土壤养分对植物-土 壤反馈过程的影响非常复杂, 受到许多因素的调 控。而随着氮沉降等因素对土壤养分含量的影响, 养分增加如何影响植物-土壤反馈并进一步影响群 落组成也是一个值得关注的问题。

综上, 某些稀有种是否可能是因为占据特殊的 生态位(例如具有特殊的养分获取策略从而占据特 殊养分含量的土壤)而难以扩大种群, 但同时又不 被常见种所淘汰? 或者是具有相对特殊的地下性 状或养分响应机制? 这是在未来值得探讨的一个 问题。

因此, 我们认为土壤养分、菌根真菌及细根性 状可以共同调控土壤负反馈, 而宿主对从枝菌根真 菌的偏好性、对外生菌根真菌的专性等因素可能也 会影响植物的分布, 从而对群落中的物种多度产生 


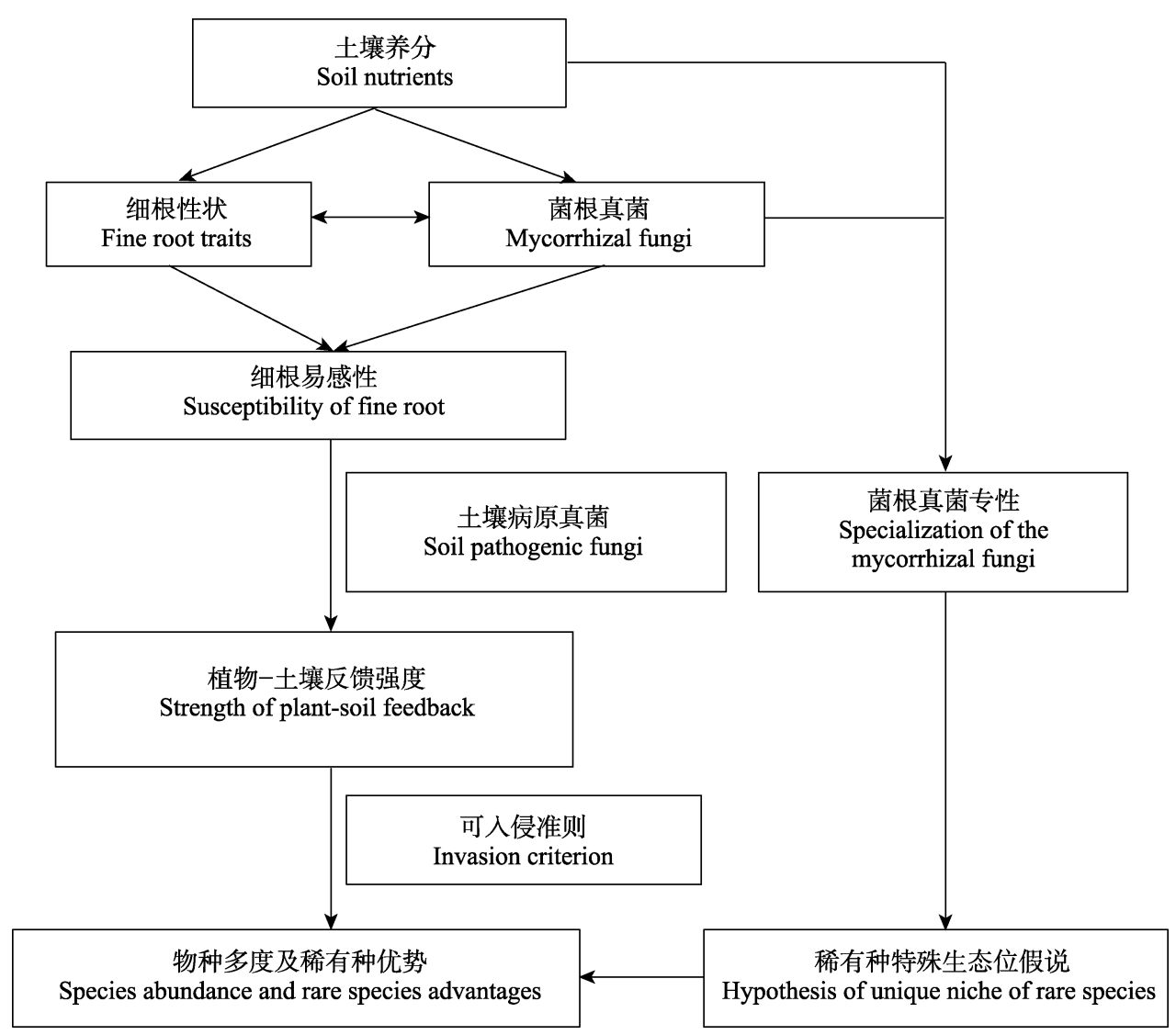

图2 植物-土壤反馈的调控因素及其对植物多度影响的框架

Fig. 2 The framework of factors formulating plant-soil feedback (PSF) and consequently determining abundance of plant species

影响(图2)。未来对土壤负反馈的研究不仅要关注病 原菌, 同时也需关注以上这些调控土壤负反馈的因 素, 以及揭示它们在稀有种维持机制方面的作用。

\section{4 其他稀有种维持的观点}

除了从土壤反馈的角度, 还有一些研究尝试从 其他角度, 如繁殖、食物网、生态位分化和竞争等 方面来解释稀有种的产生与维持机制。

Vermeij和Grosberg (2018)认为长期的稀有性是 对环境的高度适应, 他们认为稀有种对繁殖的成功 率非常依赖, 因此从繁殖的角度探讨了能使稀有种 长期维持低密度以及与繁殖相关的性状。结果发现, 自花授粉、与配偶选择有关的长距离信号传播能 力、动物辅助繁殖以及雌雄同体是有利于稀有种维 持长期稀有的性状。Bachelot等(2015)对哥斯达黎加 热带森林中负密度制约效应 10 年的动态变化进行 分析, 发现在不同年份, 稀有种还是常见种受到的 负密度制约强度更强并不一致, 而且这种变化与
气候有关一一在湿热的年份常见种受到更强的负 反馈。虽然他们并没有详细研究气候如何影响负密 度制约, 但是这一发现对解释负密度制约的变异仍 有一定参考价值。Xi等(2020)在青藏高原通过分析 高山植物散布前的种子-捕食者网络的结构特征及 其对植物种子损失率的影响发现, 不对称的拮抗网 络可以使得常见种被更多的捕食者所干扰, 使得稀 有种产生优势，进而促进物种共存。Mi等(2021)从 生态位分化的角度对森林群落中物种的生态位和 功能生态位进行研究后发现, 稀有种倾向于占据边 缘生态位而常见种则倾向于占据中心生态位, 这使 得稀有种可以避免与常见种的生态位重叠而产生 竞争; 而稀有种在功能性状上具有更大的变异, 暗 示稀有种对不同时间和空间上的资源可能有更强 的探索能力(Umaña et al, 2015)。Lloyd等(2002)对蓄 薇科和禾本科的亲缘关系相近的常见种和稀有种 进行竞争实验后发现, 植物的竞争能力与其是否稀 有并没有直接的关系。 
以上这些研究结论提示我们, 除了土壤反馈之 外的其他因素也会影响到稀有种的产生与维持, 由 于生态系统的高度复杂性, 植物-土壤反馈并非是 解释稀有种与常见种共存机制的唯一途径。例如气 候变化已被广泛认为会对生物多样性产生影响 (Bellard et al, 2012), 但这种影响对维持稀有种和常 见种的共存有何作用? 通过什么途径来产生作用? 这都是未来值得思考的问题。而繁殖失败对种群多 度的影响, 无论是对动物 (Harnik et al, 2012; Vermeij \& Grosberg, 2018)还是植物 ${ }^{\circledR}$ 都非常显著, 因此对于极度稀有物种在繁殖、定植等方面的优势 或劣势的研究也可以解释稀有种长期稀有的机制。 除此之外, 植物的系统发育信号、功能性状、种子 扩散能力以及土壤微生物等对于稀有种共存机制 的解释也有待探索。与此同时, 还应尽可能地区分 稀有种的类型, 例如是长期稀有种还是短期稀有种? 这对于提高研究结果的可靠性有重要作用。

\section{结论}

物种共存是生态学中最核心的问题之一, 而稀 有种和常见种在群落中如何共存? 稀有种的种群 受到何种限制? 它们又存在何种优势确保其不被 排除? 这些都是解释热带、亚热带森林群落高物种 多样性函待回答的问题。其中, 促进稀有种存续的 “稀有种优势” 以及限制稀有种扩大种群使其保持稀 有性的因素同样重要, 这种优势与限制如何维持其 稀有性将是未来需要重点关注的问题。

近10余年来, 随着生态学理论的发展, 新技术 与新方法不断涌现, 大量新的研究结果也带来了许 多争论, 如稀有种的“理论优势”与“实证劣势”的对 立, 这对稀有种研究而言既是挑战也是机遇。我们 希望通过对稀有种维持机制与土壤反馈理论以及 近年来的一些研究结果与争议的介绍, 提出潜在研 究方向, 以期能够吸引更多研究人员关注这一问题, 为探索稀有种的维持机制带来更多的研究动力, 进 而更好地理解植物群落的生物多样性维持机制。

致谢: 感谢百山祖国家公园管理处给予的大力支持; 感谢华东师范大学的于文波博士、陈佳乐博士以及

(1) 哀建国 (2005) 百山祖冷杉濒危机制与保护对策研究. 硕士学 位论文, 浙江大学, 杭州.
审稿人对本文提出的大量宝贵意见。

\section{ORCID}

戴冬 (D) https://orcid.org/0000-0003-2291-3487

邢华 (D) https://orcid.org/0000-0001-9501-0786

刘宇 (iD https://orcid.org/0000-0001-9869-2735

\section{参考文献}

Adler PB, HilleRisLambers J, Levine JM (2007) A niche for neutrality. Ecology Letters, 10, 95-104.

Ai D, Chu CJ, Ellwood MDF, Hou R, Wang G (2013) Migration and niche partitioning simultaneously increase species richness and rarity. Ecological Modelling, 258, 33-39.

Augspurger CK (1984) Seedling survival of tropical tree species: Interactions of dispersal distance, light-gaps, and pathogens. Ecology, 65, 1705-1712.

Augspurger CK, Kelly CK (1984) Pathogen mortality of tropical tree seedlings: Experimental studies of the effects of dispersal distance, seedling density, and light conditions. Oecologia, 61, 211-217.

Bachelot B, Kobe RK (2013) Rare species advantage? Richness of damage types due to natural enemies increases with species abundance in a wet tropical forest. Journal of Ecology, 101, 846-856.

Bachelot B, Kobe RK, Vriesendorp C (2015) Negative densitydependent mortality varies over time in a wet tropical forest, advantaging rare species, common species, or no species. Oecologia, 179, 853-861.

Bachelot B, Uriarte M, McGuire KL, Thompson J, Zimmerman J (2017) Arbuscular mycorrhizal fungal diversity and natural enemies promote coexistence of tropical tree species. Ecology, 98, 712-720.

Bagchi R, Gallery RE, Gripenberg S, Gurr SJ, Narayan L, Addis CE, Freckleton RP, Lewis OT (2014) Pathogens and insect herbivores drive rainforest plant diversity and composition. Nature, 506, 85-88.

Balzergue C, Puech-Pagès V, Bécard G, Rochange SF (2011) The regulation of arbuscular mycorrhizal symbiosis by phosphate in pea involves early and systemic signalling events. Journal of Experimental Botany, 62, 1049-1060.

Bayandala, Masaka K, Seiwa K (2017) Leaf diseases drive the Janzen-Connell mechanism regardless of light conditions: A 3-year field study. Oecologia, 183, 191-199.

Bell T, Freckleton RP, Lewis OT (2006) Plant pathogens drive density-dependent seedling mortality in a tropical tree. Ecology Letters, 9, 569-574.

Bellard C, Bertelsmeier C, Leadley P, Thuiller W, Courchamp $F$ (2012) Impacts of climate change on the future of biodiversity. Ecology Letters, 15, 365-377.

Bever JD (1999) Dynamics within mutualism and the 
maintenance of diversity: Inference from a model of interguild frequency dependence. Ecology Letters, 2, 52-61.

Bever JD (2002) Negative feedback within a mutualism: Host-specific growth of mycorrhizal fungi reduces plant benefit. Proceedings of the Royal Society B: Biological Sciences, 269, 2595-2601.

Branscheid A, Sieh D, Pant BD, May P, Devers EA, Elkrog A, Schauser L, Scheible WR, Krajinski F (2010) Expression pattern suggests a role of MiR399 in the regulation of the cellular response to local $\mathrm{Pi}$ increase during arbuscular mycorrhizal symbiosis. Molecular Plant Microbe Interactions, 23, 915-926.

Brown JH (1984) On the relationship between abundance and distribution of species. The American Naturalist, 124, 255279.

Brundrett MC (2009) Mycorrhizal associations and other means of nutrition of vascular plants: Understanding the global diversity of host plants by resolving conflicting information and developing reliable means of diagnosis. Plant and Soil, 320, 37-77.

Cameron DD, Neal AL, van Wees SCM, Ton J (2013) Mycorrhiza-induced resistance: More than the sum of its parts? Trends in Plant Science, 18, 539-545.

Caughley G (1994) Directions in conservation biology. Journal of Animal Ecology, 63, 215-244.

Chapman ASA, Tunnicliffe V, Bates AE (2018) Both rare and common species make unique contributions to functional diversity in an ecosystem unaffected by human activities. Diversity and Distributions, 24, 568-578.

Chen L, Swenson NG, Ji NN, Mi XC, Ren HB, Guo LD, Ma KP (2019) Differential soil fungus accumulation and density dependence of trees in a subtropical forest. Science, 366, 124-128.

Chesson P (2000) Mechanisms of maintenance of species diversity. Annual Review of Ecology and Systematics, 31, 343-366.

Chesson P (2008) Quantifying and testing species coexistence mechanisms. In: Unity in Diversity: Reflections on Ecology after the Legacy of Ramon Margalef (eds Valladares F, Camacho A, Elosegui A, Gracia C, Estrada M, Senar JC, Gili JM), pp. 119-164. BBVA, Bilbao, Spain.

Chu CJ, Wang YS, Liu Y, Jiang L, He FL (2017) Advances in species coexistence theory. Biodiversity Science, 25, 345-354. (in Chinese with English abstract) [储诚进, 王西 石, 刘宇, 蒋林, 何芳良 (2017) 物种共存理论研究进展. 生物多样性, 25, 345-354.]

Comita LS, Muller-Landau HC, Aguilar S, Hubbell SP (2010) Asymmetric density dependence shapes species abundances in a tropical tree community. Science, 329, 330-332.

Connell JH (1971) On the role of natural enemies in preventing competitive exclusion in some marine animals and in rain forest trees. In: Dynamics of Populations (eds Boer PJD, Gradwell GR), pp. 298-312. Center for Agriculture
Publishing and Documentation, Wageningen.

Connell JH, Tracey JG, Webb LJ (1984) Compensatory recruitment, growth, and mortality as factors maintaining rain forest tree diversity. Ecological Monographs, 54, 141-164.

Corrales A, Mangan SA, Turner BL, Dalling JW (2016) An ectomycorrhizal nitrogen economy facilitates monodominance in a neotropical forest. Ecology Letters, 19, 383-392.

Cortois R, Schröder-Georgi T, Weigelt A, van der Putten WH, De Deyn GB (2016) Plant-soil feedbacks: Role of plant functional group and plant traits. Journal of Ecology, 104, 1608-1617.

Dawson W, Fischer M, van Kleunen M (2012) Common and rare plant species respond differently to fertilisation and competition, whether they are alien or native. Ecology Letters, 15, 873-880.

Eissenstat DM (1992) Costs and benefits of constructing roots of small diameter. Journal of Plant Nutrition, 15, 763-782.

Enquist BJ, Feng X, Boyle B, Maitner B, Newman EA, Jørgensen PM, Roehrdanz PR, Thiers BM, Burger JR, Corlett RT, Couvreur TLP, Dauby G, Donoghue JC, Foden W, Lovett JC, Marquet PA, Merow C, Midgley G, Morueta-Holme N, Neves DM, Oliveira-Filho AT, Kraft NJB, Park DS, Peet RK, Pillet M, Serra-Diaz JM, Sandel B, Schildhauer M, Šímová I, Violle C, Wieringa JJ, Wiser SK, Hannah L, Svenning JC, McGill BJ (2019) The commonness of rarity: Global and future distribution of rarity across land plants. Science Advances, 5, eaaz0414.

Forrister DL, Endara MJ, Younkin GC, Coley PD, Kursar TA (2019) Herbivores as drivers of negative density dependence in tropical forest saplings. Science, 363, 1213-1216.

Gaston KJ, Blackburn TM, Lawton JH (1997) Interspecific abundance-range size relationships: An appraisal of mechanisms. Journal of Animal Ecology, 66, 579-601.

Grainger TN, Levine JM, Gilbert B (2019) The invasion criterion: A common currency for ecological research. Trends in Ecology \& Evolution, 34, 925-935.

Grinnell J (1917) The niche-relationships of the California thrasher. The Auk, 34, 427-433.

Guo DL, Xia MX, Wei X, Chang WJ, Liu Y, Wang ZQ (2008) Anatomical traits associated with absorption and mycorrhizal colonization are linked to root branch order in twenty-three Chinese temperate tree species. New Phytologist, 180, 673-683.

Gustafson DJ, Casper BB (2004) Nutrient addition affects AM fungal performance and expression of plant/fungal feedback in three serpentine grasses. Plant and Soil, 259, 9-17.

Harnik PG, Simpson C, Payne JL (2012) Long-term differences in extinction risk among the seven forms of rarity. Proceedings of the Royal Society B: Biological Sciences, 279, 4969-4976.

Hart TB, Hart JA, Murphy PG (1989) Monodominant and 
species-rich forests of the humid tropics: Causes for their co-occurrence. The American Naturalist, 133, 613-633.

He FL (2009) Price of prosperity: Economic development and biological conservation in China. Journal of Applied Ecology, 46, 511-515.

Ho MD, Rosas JC, Brown KM, Lynch JP (2005) Root architectural tradeoffs for water and phosphorus acquisition. Functional Plant Biology, 32, 737-748.

Hubbell SP, Foster RB (1986) Canopy gaps and the dynamics of a Neotropical forest. In: Plant Ecology (ed. Crawley MJ), pp. 77-96. Blackwell, Oxford.

Hyatt LA, Rosenberg MS, Howard TG, Bole G, Fang W, Anastasia J, Brown K, Grella R, Hinman K, Kurdziel JP, Gurevitch J (2003) The distance dependence prediction of the Janzen-Connell hypothesis: A meta-analysis. Oikos, 103, 590-602.

in't Zandt D, van den Brink A, de Kroon H, Visser EJW (2019) Plant-soil feedback is shut down when nutrients come to town. Plant and Soil, 439, 541-551.

Jain M, Flynn DFB, Prager CM, Hart GM, DeVan CM, Ahrestani FS, Palmer MI, Bunker DE, Knops JMH, Jouseau CF, Naeem S (2014) The importance of rare species: A trait-based assessment of rare species contributions to functional diversity and possible ecosystem function in tall-grass prairies. Ecology and Evolution, 4, 104-112.

Janzen DH (1970) Herbivores and the number of tree species in tropical forests. The American Naturalist, 104, 501-528.

Jia SH, Wang XG, Yuan ZQ, Lin F, Ye J, Lin GG, Hao ZQ, Bagchi R (2020) Tree species traits affect which natural enemies drive the Janzen-Connell effect in a temperate forest. Nature Communications, 11, 286.

Johnson DJ, Beaulieu WT, Bever JD, Clay K (2012) Conspecific negative density dependence and forest diversity. Science, 336, 904-907.

Jung SC, Martinez-Medina A, Lopez-Raez JA, Pozo MJ (2012) Mycorrhiza-induced resistance and priming of plant defenses. Journal of Chemical Ecology, 38, 651-664.

Keane RM, Crawley MJ (2002) Exotic plant invasions and the enemy release hypothesis. Trends in Ecology \& Evolution, 17, 164-170.

Kempel A, Rindisbacher A, Fischer M, Allan E (2018) Plant soil feedback strength in relation to large-scale plant rarity and phylogenetic relatedness. Ecology, 99, 597-606.

Klironomos JN (2002) Feedback with soil biota contributes to plant rarity and invasiveness in communities. Nature, 417, 67-70.

Klironomos JN (2003) Variation in plant response to native and exotic arbuscular mycorrhizal fungi. Ecology, 84, 22922301.

Kobae Y, Ohmori Y, Saito C, Yano K, Ohtomo R, Fujiwara T (2016) Phosphate treatment strongly inhibits new arbuscule development but not the maintenance of arbuscule in mycorrhizal rice roots. Plant Physiology, 171, 566-579.
Kos M, Veendrick J, Bezemer TM (2013) Local variation in conspecific plant density influences plant-soil feedback in a natural grassland. Basic and Applied Ecology, 14, 506-514.

Kramer-Walter KR, Bellingham PJ, Millar TR, Smissen RD, Richardson SJ, Laughlin DC (2016) Root traits are multidimensional: Specific root length is independent from root tissue density and the plant economic spectrum. Journal of Ecology, 104, 1299-1310.

Laliberté E, Lambers H, Burgess TI, Wright SJ (2015) Phosphorus limitation, soil-borne pathogens and the coexistence of plant species in hyperdiverse forests and shrublands. New Phytologist, 206, 507-521.

Laliberté E, Turner BL, Costes T, Pearse SJ, Wyrwoll KH, Zemunik G, Lambers H (2012) Experimental assessment of nutrient limitation along a 2-million-year dune chronosequence in the south-western Australia biodiversity hotspot. Journal of Ecology, 100, 631-642.

Le Bagousse-Pinguet Y, Gross N, Saiz H, Maestre FT, Ruiz S, Dacal M, Asensio S, Ochoa V, Gozalo B, Cornelissen JHC, Deschamps L, García C, Maire V, Milla R, Salinas N, Wang JT, Singh BK, García-Palacios P (2021) Functional rarity and evenness are key facets of biodiversity to boost multifunctionality. Proceedings of the National Academy of Sciences, USA, 118, e2019355118.

Leimu R, Mutikainen P, Koricheva J, Fischer M (2006) How general are positive relationships between plant population size, fitness and genetic variation? Journal of Ecology, 94, 942-952.

Leitão RP, Zuanon J, Villéger S, Williams SE, Baraloto C, Fortunel C, Mendonça FP, Mouillot D (2016) Rare species contribute disproportionately to the functional structure of species assemblages. Proceedings of the Royal Society B: Biological Sciences, 283, 20160084.

Li RB, Yu SX, Wang YF, Staehelin C, Zang RG (2009) Distance-dependent effects of soil-derived biota on seedling survival of the tropical tree legume Ormosia semicastrata. Journal of Vegetation Science, 20, 527-534.

Liang MX, Liu XB, Etienne RS, Huang FM, Wang YF, Yu SX (2015) Arbuscular mycorrhizal fungi counteract the JanzenConnell effect of soil pathogens. Ecology, 96, 562-574.

Liang MX, Liu XB, Gilbert GS, Zheng Y, Luo S, Huang FM, Yu SX (2016) Adult trees cause density-dependent mortality in conspecific seedlings by regulating the frequency of pathogenic soil fungi. Ecology Letters, 19, 1448-1456.

Liu Y, Yu SX, Xie ZP, Staehelin C (2012) Analysis of a negative plant-soil feedback in a subtropical monsoon forest. Journal of Ecology, 100, 1019-1028.

Liu Y, Fang SQ, Chesson P, He FL (2015) The effect of soil-borne pathogens depends on the abundance of host tree species. Nature Communications, 6, 10017.

Liu Y, He FL (2019) Incorporating the disease triangle framework for testing the effect of soil-borne pathogens on tree species diversity. Functional Ecology, 33, 1211-1222. 
Lloyd KM, Lee WG, Wilson JB (2002) Competitive abilities of rare and common plants: Comparisons using Acaena (Rosaceae) and Chionochloa (Poaceae) from New Zealand. Conservation Biology, 16, 975-985.

Lynch JP, Ho MD (2005) Rhizoeconomics: Carbon costs of phosphorus acquisition. Plant and Soil, 269, 45-56.

MacArthur R, Levins R (1967) The limiting similarity, convergence, and divergence of coexisting species. The American Naturalist, 101, 377-385.

MacArthur R (1972) Geographical Ecology. Princeton University Press, Princeton.

MacDougall AS, Rillig MC, Klironomos JN (2011) Weak conspecific feedbacks and exotic dominance in a speciesrich savannah. Proceedings of the Royal Society B: Biological Sciences, 278, 2939-2945.

Mangan SA, Herre EA, Bever JD (2010a) Specificity between Neotropical tree seedlings and their fungal mutualists leads to plant-soil feedback. Ecology, 91, 2594-2603.

Mangan SA, Schnitzer SA, Herre EA, Mack KML, Valencia MC, Sanchez EI, Bever JD (2010b) Negative plant-soil feedback predicts tree-species relative abundance in a tropical forest. Nature, 466, 752-755.

Marden JH, Mangan SA, Peterson MP, Wafula E, Fescemyer HW, Der JP, dePamphilis CW, Comita LS (2017) Ecological genomics of tropical trees: How local population size and allelic diversity of resistance genes relate to immune responses, cosusceptibility to pathogens, and negative density dependence. Molecular Ecology, 26, 24982513.

Maron JL, Laney Smith A, Ortega YK, Pearson DE, Callaway RM (2016) Negative plant-soil feedbacks increase with plant abundance, and are unchanged by competition. Ecology, 97, 2055-2063.

Marx DH (1972) Ectomycorrhizae as biological deterrents to pathogenic root infections. Annual Review of Phytopathology, 10, 429-454.

McCormack ML, Dickie IA, Eissenstat DM, Fahey TJ, Fernandez CW, Guo DL, Helmisaari HS, Hobbie EA, Iversen CM, Jackson RB, Leppälammi-Kujansuu J, Norby RJ, Phillips RP, Pregitzer KS, Pritchard SG, Rewald B, Zadworny M (2015) Redefining fine roots improves understanding of below-ground contributions to terrestrial biosphere processes. New Phytologist, 207, 505-518.

McGuire KL (2007) Common ectomycorrhizal networks may maintain monodominance in a tropical rain forest. Ecology, 88, 567-574.

Mi XC, Sun ZH, Song YF, Liu XJ, Yang J, Wu JJ, Ci XQ, Li J, Lin LX, Cao M, Ma KP (2021) Rare tree species have narrow environmental but not functional niches. Functional Ecology, 35, 511-520.

Mi XC, Swenson NG, Valencia R, Kress WJ, Erickson DL, Pérez ÁJ, Ren HB, Su SH, Gunatilleke N, Gunatilleke S, Hao ZQ, Ye WH, Cao M, Suresh HS, Dattaraja HS,
Sukumar R, Ma KP (2012) The contribution of rare species to community phylogenetic diversity across a global network of forest plots. The American Naturalist, 180, E17-E30.

Newsham KK, Fitter AH, Watkinson AR (1995) Arbuscular mycorrhiza protect an annual grass from root pathogenic fungi in the field. Journal of Ecology, 83, 991-1000.

Packer A, Clay K (2000) Soil pathogens and spatial patterns of seedling mortality in a temperate tree. Nature, 404, 278-281.

Parker IM, Saunders M, Bontrager M, Weitz AP, Hendricks R, Magarey R, Suiter K, Gilbert GS (2015) Phylogenetic structure and host abundance drive disease pressure in communities. Nature, 520, 542-544.

Petermann JS, Fergus AJF, Turnbull LA, Schmid B (2008) Janzen-Connell effects are widespread and strong enough to maintain diversity in grasslands. Ecology, 89, 2399-2406.

Pimm SL, Jones HL, Diamond J (1988) On the risk of extinction. The American Naturalist, 132, 757-785.

Pimm SL, Russell GJ, Gittleman JL, Brooks TM (1995) The future of biodiversity. Science, 269, 347-350.

Plassard C, Dell B (2010) Phosphorus nutrition of mycorrhizal trees. Tree Physiology, 30, 1129-1139.

Porter SS, Sachs JL (2020) Agriculture and the disruption of plant-microbial symbiosis. Trends in Ecology \& Evolution, 35, 426-439.

Pregitzer KS (2002) Fine roots of trees-A new perspective. New Phytologist, 154, 267-270.

Preston FW (1962) The canonical distribution of commonness and rarity: Part I. Ecology, 43, 185-215.

Rabinowitz D (1981) Seven forms of rarity. In: The Biological Aspects of Rare Plant Conservation (ed. Synge H), pp. 205-217. John Wiley \& Sons, Chichester.

Regus JU, Wendlandt CE, Bantay RM, Gano-Cohen KA, Gleason NJ, Hollowell AC, O’Neill MR, Shahin KK, Sachs JL (2017) Nitrogen deposition decreases the benefits of symbiosis in a native legume. Plant and Soil, 414, 159-170.

Reich PB (2014) The world-wide 'fast-slow' plant economics spectrum: A traits manifesto. Journal of Ecology, 102, 275-301.

Rousseau JVD, Sylvia DM, Fox AJ (1994) Contribution of ectomycorrhiza to the potential nutrient-absorbing surface of pine. New Phytologist, 128, 639-644.

Schroeder JW, Dobson A, Mangan SA, Petticord DF, Herre EA (2020) Mutualist and pathogen traits interact to affect plant community structure in a spatially explicit model. Nature Communications, 11, 2204.

Schroeder JW, Martin JT, Angulo DF, Barbosa JM, Perea R, Arias-Del Razo I, Sebastián-González E, Dirzo R (2018) Community composition and diversity of Neotropical rootassociated fungi in common and rare trees. Biotropica, 50, 694-703.

Siepielski AM, McPeek MA (2010) On the evidence for species coexistence: A critique of the coexistence program. 
Ecology, 91, 3153-3164.

Smith MD, Knapp AK (2003) Dominant species maintain ecosystem function with non-random species loss. Ecology Letters, 6, 509-517.

Smith SE, Read DJ (1997) Mycorrhizal Symbioses. Academic Press, London.

Stump SM, Marden JH, Beckman NG, Mangan SA, Comita LS (2020) Resistance genes affect how pathogens maintain plant abundance and diversity. The American Naturalist, 196, 472-486.

Umaña MN, Zhang CC, Cao M, Lin LX, Swenson NG (2015) Commonness, rarity, and intraspecific variation in traits and performance in tropical tree seedlings. Ecology Letters, 18, 1329-1337.

van de Voorde TFJ, van der Putten WH, Bezemer TM (2012) The importance of plant-soil interactions, soil nutrients, and plant life history traits for the temporal dynamics of Jacobaea vulgaris in a chronosequence of old-fields. Oikos, 121, 1251-1262.

van der Heijden MGA, Boller T, Wiemken A, Sanders IR (1998) Different arbuscular mycorrhizal fungal species are potential determinants of plant community structure. Ecology, 79, 2082-2091.

Vermeij GJ, Grosberg RK (2018) Rarity and persistence. Ecology Letters, 21, 3-8.

Wehner J, Antunes PM, Powell JR, Mazukatow J, Rillig MC (2010) Plant pathogen protection by arbuscular mycorrhizas: A role for fungal diversity? Pedobiologia, 53, 197-201.

Xi XQ, Yang YHS, Tylianakis JM, Yang SH, Dong YR, Sun
SC (2020) Asymmetric interactions of seed-predation network contribute to rare-species advantage. Ecology, 101, e03050.

Yenni G, Adler PB, Morgan Ernest SK (2012) Strong selflimitation promotes the persistence of rare species. Ecology, 93, 456-461.

Yenni G, Adler PB, Morgan Ernest SK (2017) Do persistent rare species experience stronger negative frequency dependence than common species? Global Ecology and Biogeography, 26, 513-523.

$\mathrm{Yu}$ WB, Li SP (2020) Modern coexistence theory as a framework for invasion ecology. Biodiversity Science, 28, 1362-1375. (in Chinese with English abstract) [于文波, 黎 绍鹏 (2020) 基于现代物种共存理论的入侵生态学概念 框架. 生物多样性, 28, 1362-1375.]

Zemunik G, Turner BL, Lambers H, Laliberté E (2015) Diversity of plant nutrient-acquisition strategies increases during long-term ecosystem development. Nature Plants, 1, 15050.

Zhang ZJ, van Kleunen M (2019) Common alien plants are more competitive than rare natives but not than common natives. Ecology Letters, 22, 1378-1386.

Zhu Y, Mi XC, Ma KP (2009) A mechanism of plant species coexistence: The negative density-dependent hypothesis. Biodiversity Science, 17, 594-604. (in Chinese with English abstract) [祝燕, 米湘成, 马克平 (2009) 植物群落物种共 存机制: 负密度制约假说. 生物多样性, 17, 594-604.]

(责任编委: 米湘成 责任编辑: 黄祥忠) 